\title{
Postembedding immunocytochemistry of large sections of brain tissue: an improved flat-embedding technique
}

\author{
David T. Lanue ${ }^{*}$, Jeffery A. Winer \\ Division of Neurobiology, Department of Molecular and Cell Biology, University of California at Berkeley, Berkeley, CA 94720-3200, U/SA
}

Received 26 May 1995; accepted 8 August 1995

\begin{abstract}
A method for osmicating, dehydrating, and flat-embedding large slabs of brain tissue in epoxy resin is presented. This permits the production of semithin sections for postembedding immunocytochemistry that are far larger than can be obtained with other embedding approaches. Vibratomed slabs, 50-200 $\mu \mathrm{m}$ thick and as large as $6 \times 8 \mathrm{~mm}$ are embedded in a 'soft' Araldite epoxy. The slabs are laminated onto the flat surface of a pre-cast epoxy slide. After polymerization, the tissue can be studied on the slide as a whole mount to view osmicated fiber tracts, or in experimental tract-tracing studies, to locate retrogradely labeled cells before semithin sections are cut. The rigidity of the epoxy slide ensures that the slabs remain flat and are easily removed and mounted for resectioning. Semithin sections are cut using $8 \mathrm{~mm}$ wide glass knives or a $6 \mathrm{~mm}$ wide diamond knife and are mounted singly or in serial pairs and immunostained using conventional etching and immunoperoxidase techniques. The relative softness of the epoxy permits dozens of semithin sections to be cut from large blocks without appreciably degrading a glass knife edge. After further polymerization the embedment is also compatible with electron microscopy.
\end{abstract}

Keywords: Colocalization; Araldite; Semithin section; Epoxy; GABA; Glycine; Wholemount

\section{Introduction}

Epoxy embedments of osmicated tissue provide superior preservation of morphological detail (Hayat, 1970). While this method is essential for ultrastructural study, it can also provide a valuable resource for high-resolution light microscopy. Postembedding immunocytochemistry uses an etching process to dissolve the epoxy embedment from semithin (1-2 $\mu \mathrm{m}$ thick) sections, allowing antisera access to the tissue, and thus extends this resolution to the localization of tissue antigens (Erlandsen et al., 1979). For example, staining serial semithin sections can reveal cellular colocalization of multiple neurotransmitter molecules (Yingcharoen et al., 1989; Winer et al., 1995) and, in tract tracing experiments, neurotransmitters and retrograde labeling can be colocalized without ambiguity (Saint Marie et al., 1989). An impediment to the potential of the postembedding technique as a more general tool in neuroanatomy is the comparatively small size of the semithin

\footnotetext{
*Corresponding author: Tel.: (510) 642-9637; Fax: (510) 643-6791; E-mail: dtlane@uclink.berkeley.edu.
}

sections that can typically be prepared. This is due primarily to the difficulty of achieving flat embedments of large blocks of brain tissue, as well as the challenges of sectioning large block faces. Distortion of the tissue during dehydration is a primary obstacle to preparing large area embedments. When embedding Vibratomed sections (slabs) that are relatively thick $(300-500 \mu \mathrm{m})$ and less than approximately $2 \times 3 \mathrm{~mm}$ on a side, this distortion is rarely evident. However, for sections up to approximately $6 \times 8$ $\mathrm{mm}$ in size and thin enough to be useful for flat-embedding $(50-200 \mu \mathrm{m})$, this distortion is pronounced and can result in a saddle-shaped slab that may fracture during flattening. The second challenge is the physical handling of the tissue during embedding to ensure that the section has a truly flat face available at the surface of the polymerized block.

Several flat-embedding methods for light and/or electron microscopy utilize a sandwich of tissue between flat layers or surfaces. An early, pre-Vibratome technique for embedding large sections used razor-sliced, osmicated blocks embedded in softened epoxy. They were sectioned on a sliding microtome for light microscopic study, after which areas were chosen for re-embedment between cover- 
slips and resectioned for electron microscopy (Grimley, 1965). In a similar approach, osmicated blocks were sectioned with a sliding steel knife and flat-embedded in
Araldite epoxy between acetate foils (Holländer, 1970). More recently, Vibratomed tissue slabs have been placed between Teflon ${ }^{\mathrm{TM}}$-coated slides and coverslips (Wilson and
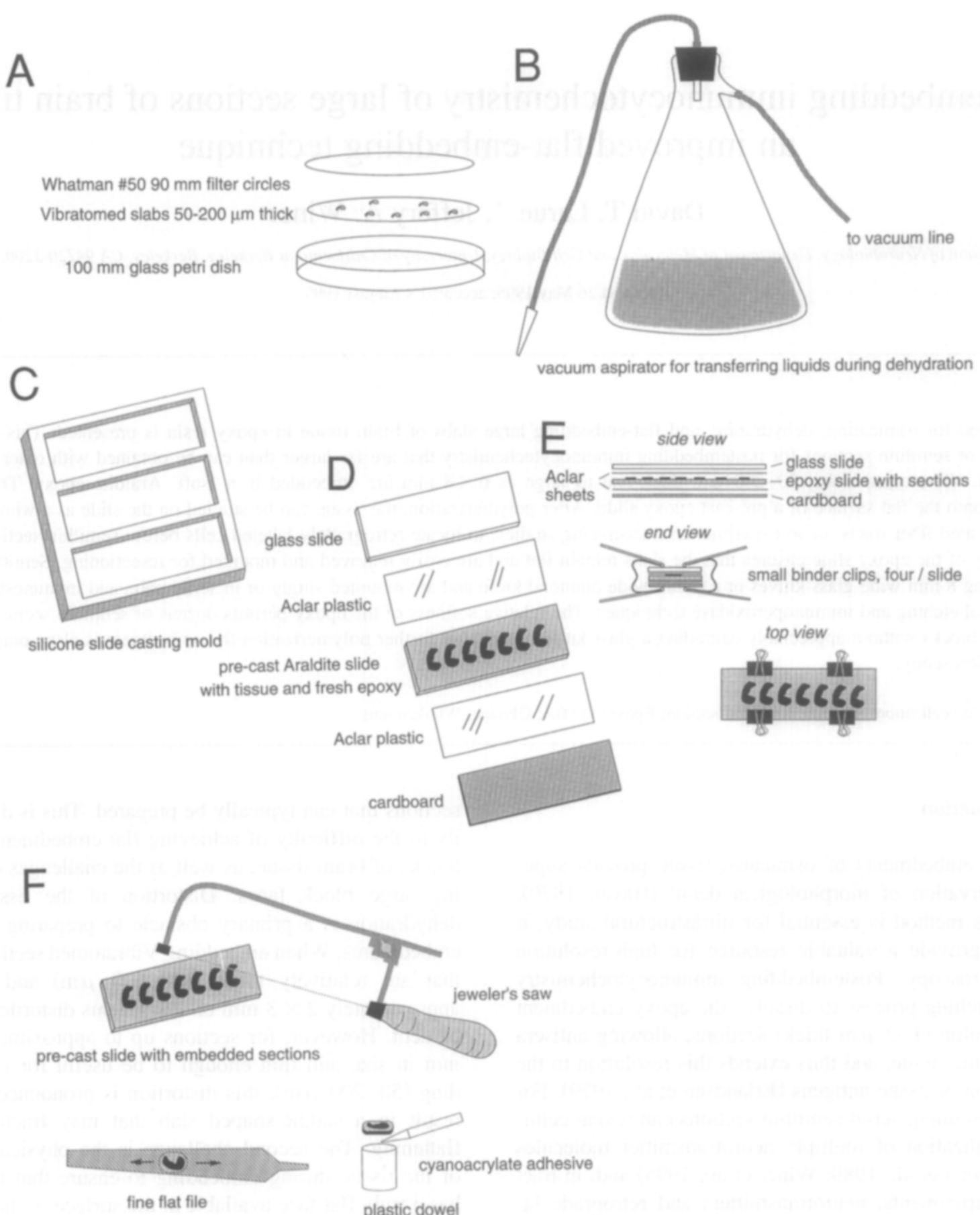

Fig. 1. Schematic illustration of the embedding sequence. A: The osmication, dehydration, and epoxy infiltration process is carried out with the sections sandwiched between the filter paper circles. This minimizes handling of the tissue and reduces curling or distorting during the dehydration. B: The vacuum aspirator facilitates the transfer of liquids during osmication and dehydration; the vapor pressure of propylene oxide is too high and it must be decanted. $\mathrm{C}$ : Epoxy slides are cast in advance in a silicone mold. D: After the slabs are infiltrated with epoxy, they are transferred to the flat surface of the cast slide (with a minimum amount of resin) and the embedding sandwich is assembled. E: Small binder clips compress the sandwich, ensuring that the surface of the tissue conforms to the plane defined by the glass slide during polymerization. F: After polymerization, the sandwich separates readily because of the Aclar plastic layers. The embedded blocks are then recovered by holding the slide in a vise and cutting them out with a jeweler's fine saw. The bottom of the block should be filed flat to form a uniform bonding surface before attachment to a plastic dowel with cyanoacrylate adhesive. The block may now be trimmed and sectioned. Typical embedments are flat enough that less than $10 \mu \mathrm{m}$ must be cut to expose a full block face. 
Groves, 1979), between nylon mesh and a BEEM ${ }^{\mathrm{TM}}$ capsule cap (Schwartz, 1982), between sheets of $\mathrm{Aclar}^{\mathrm{TM}}$ plastic (Anthony and King, 1986; Hancock, 1986), or polystyrene layers (Papka et al., 1986), or between acetate sheets and glass slides (Yu and Schwartz, 1989).

Each of these methods, while producing acceptable slab flatness, requires careful handling to separate the plastic layers or coverslips and retrieve the rather thin and fragile embedment, which must then be trimmed and mounted for resectioning. The present approach minimizes tissue distortion and handling of the sections and uses a cast epoxy slide as a substrate for the embedment. Briefly, tissue slabs were osmicated, dehydrated, then infiltrated in epoxy while held between two filter paper circles in a petri dish. They were placed on the flat surface of a pre-cast epoxy slide, covered with a layer of Aclar and a glass slide on top, then clamped together and polymerized. The rigid epoxy substrate offers several advantages, such as dimensional stability, easy retrieval and mounting of the specimens, and the prospect of generating very large semithin sections, even from $50 \mu \mathrm{m}$ thick embedments. The slabs can be studied or photographed in situ and can be removed easily with a jeweler's saw and mounted for further sectioning. This method permits the routine embedding of sections (up to $6 \times 8 \mathrm{~mm}$ and $50-200 \mu \mathrm{m}$ thick), and it assures flatness and ease of mounting for subsequent semithin resectioning and postembedding immunocytochemistry. A modified formula for 'soft' Araldite epoxy allows dozens of semithin sections from these large blocks to be cut with minimal glass knife wear, reducing knife changes and subsequent refacing of the block. Sections cut from the soft epoxy are also easier to deplasticize or 'etch' for postembedding than those using conventional Epon-Araldite recipes.

\section{Materials and methods}

\section{2.l. Fixation}

Tissue was fixed for immunocytochemical localization of small molecule antigen conjugates such as amino acid neurotransmitters. A transcardial perfusion of mixed aldehydes, usually $2-4 \%$ paraformaldehyde and $0.25-2.5 \%$ glutaraldehyde, followed a brief wash with phosphatebuffered saline (PBS); the specific concentration and ratio of fixatives depended on the nature of the experiment. Postfixation for $1-3 \mathrm{~h}$ followed. The tissue was then placed in $6 \%$ sucrose in $0.12 \mathrm{M}$ phosphate buffer (PB) at $4^{\circ} \mathrm{C}$ overnight or was sectioned immediately.

\subsection{Vibratome sectioning}

Slabs 50-200 $\mu \mathrm{m}$ thick were cut and collected in cold $0.12 \mathrm{M}$ PB, pH 7.4. Thinner slabs $(\leq 100 \mu \mathrm{m})$ are recommended for tract-tracing histochemistry to allow thorough penetration of the substrate and chromagen reagents. Thinner slabs are also preferable if transillumination and/or photomicrography or camera-lucida drawing of the slab is required before resectioning, since thicker material may become too opaque after osmication.

\subsection{Osmication}

Osmication and dehydration steps were carried out in $100 \mathrm{~mm}$ glass petri dishes with the slabs arranged between $90 \mathrm{~mm}$ hardened filter paper circles (Whatman \#50) (Fig. 1A). A filter paper circle was placed in the petri dish and moistened with a few milliliters of PB. The sections were transferred to the dish with a camel's hair brush and unfolded and oriented. A second paper circle was carefully placed over the sections; the surface tension between the circles maintained the flatness and position of the sections. The buffer was removed and replaced with $0.5-1.0 \%$ $\mathrm{OsO}_{4}$ in $\mathrm{PB}$ for 30-90 min, depending on the section thickness. A vacuum aspirator with a fluid trap bottle was used to transfer the fluids without moving the sections (Fig. 1B). Osmication stabilizes the lipid fraction of the tissue and reduces differential shrinkage and curling, while the staining facilitates handling the otherwise transparent tissue during embedding.

\subsection{Dehydration and infiltration}

After osmication, the tissue was rinsed $(3 \times 5 \mathrm{~min}$ in $\mathrm{PB})$, then dehydrated through ascending ethanols (EtOH) $(40 \%, 50 \%, 70 \% \times 2,80 \%, 95 \%, 5$ min each), followed by $100 \% \mathrm{EtOH}$ and propylene oxide $(2 \times 5 \mathrm{~min}$ each). After changing to propylene oxide/epoxy $(1: 1$, see formula below) the slabs were infiltrated overnight in an air-tight chamber. The dilute epoxy was then removed and the infiltration was completed (fresh $100 \%$ plastic for $3 \mathrm{~h}$ ).

\subsection{Epoxy ('soft' formula)}

This Araldite mixture is hard enough for sectioning in the semithin range but sufficiently soft to minimize damage to the glass knives. This is achieved by the addition of $10 \%$ of the plasticizer, dibutyl phthalate (after Grimley, 1965).

Araldite 6005

Dodecenyl succinic anhydride (DDSA)

Dibutyl phthalate (DBP)

Blend then add the catalyst:

Benzyldimethylamine (BDMA)
$23 \mathrm{~g}$

$20.5 \mathrm{~g}$

$5 \mathrm{~g}$

$0.75 \mathrm{~g}$
Resin components available in Araldite 6005 kit (Electron Microscopy Sciences Inc., P.O. Box 251, Fort Washington, PA 19034, USA; cat. \#13920).

\subsection{Embedding}

After being transferred through a last change of fresh epoxy, the slabs were trimmed and oriented for the final embedding, often with the aid of a transilluminating stere- 
omicroscope. Cast epoxy slides were made in advance in a $1 \times 3^{\prime \prime}$ silicone Giammara-Hanker 'Cast-a-slide' mold (Energy Beam Sciences Inc., P.O. Box 467, Agawam, MA 01001, USA; cat. \#MR-3-1) (Fig. 1C). The slabs were placed, along with a small amount of fresh resin, on the flat surface (originally the bottom, cast surface) of the epoxy slide. A piece of Aclar ${ }^{\mathrm{TM}}$ plastic film (Allied Chemical Inc., Ted Pella Inc., P.O. Box 492477, Redding, CA 96049-2477, USA; cat. \# 10502) covered the slabs, and a glass slide was placed on top (see Fig. 1D). Another Aclar strip was placed below the epoxy slide and, beneath this, a piece of cardboard formed the bottom layer. The entire sandwich was clamped together with four small binder clips (Fig. 1E). The cardboard conformed to the uneven (meniscal) surface of the cast epoxy slide and transferred even pressure against the flat glass slide at the top of the sandwich. The slide sandwiches were polymerized at $60^{\circ} \mathrm{C}$ for 16-18 h. Higher temperatures or longer polymerization may result in a block that is excessively hard and difficult to section with glass knives.

\subsection{Mounting}

After the slide sandwiches cooled, the clips were removed; the Aclar strips allow the layers to separate easily. The slabs can then be studied and/or photographed directly as whole mounts (especially those $\leq 100 \mu \mathrm{m}$ thick). Slabs chosen for resectioning were removed from the slide with a jeweler's saw and mounted on acrylic cylinders (Ted Pella Inc., cat. \#10580) with cyanoacrylate adhesive (Fig. 1F). The bottom (meniscal) surface of the cast slide is slightly concave and was filed flat before mounting.

\subsection{Microtomy}

Semithin sections $(0.5-2 \mu \mathrm{m})$ were cut on an ultramicrotome (LKB Ultratome III), with $8 \mathrm{~mm}$ wide glass knives fitted with plastic boats (Truffs ${ }^{\mathrm{TM}}$; Energy Beam Sciences Inc.; cat. \#840045) or a $6 \mathrm{~mm}$ diamond 'Histo'Knife (Diatome Inc., P.O. Box 125, Fort Washington, PA 19034, USA). The sections were retrieved from the water with an angled, fire-polished glass pipet, transferred to a bead of filtered, deionized water on a clean glass slide, and heat annealed at $90^{\circ} \mathrm{C}$ on a hot plate until flat.

\subsection{Postembedding protocol}

(1) The sections were circled with a hydrophobic ring using a 'PAP-Pen' (Daido Sangyo, Japan; Ted Pella Inc., cat. \#22304). This keeps immunoreagents pooled over the section during the incubations. Ten slides were placed in a glass staining rack for use in small staining dishes (up to 19 slides will fit in staircase fashion).

(2) Etching: slides were placed in saturated ethanolic sodium hydroxide etching solution $(\sim 10 \% \mathrm{NaOH}$ in absolute EtOH; see Lane and Europa, 1965) for 120 min.

Prepare etching solution 2-3 days in advance; it will turn honey-colored when ready to use. Caution: sections must not be allowed to dry out after etching or antigenicity will be lost
(3) Rinse: $100 \%$ EtOH
$1 \min$
(4) Neutralize: $1 \%$ glacial acetic acid $1 \mathrm{~min}$ in $100 \% \mathrm{EtOH}$
(5) Rinse: $100 \%$ EtOH $1 \mathrm{~min}$
(6) De-osmicate: $0.3 \% \mathrm{H}_{2} \mathrm{O}_{2}$ in $15 \mathrm{~min}$ $100 \% \mathrm{EtOH}$

(7) Rehydration: $95 \%, 70 \%, 50 \%, \quad 1$ min each 20\% EtOH, $0.01 \mathrm{M}$ PBS

(8) Blocking serum: $5 \%$ normal serum $60 \mathrm{~min}$ of the species of the second or 'bridge' antibody in PBS. For rabbit primary antisera, the blocking serum was goat.

(9) Incubation: The antiserum dilutions were prepared in the blocking serum; e.g., rabbit anti- overnight at room temperature normal goat serum. The antiserum was applied as a bubble over the section with the slide lying horizontally in a humid chamber.

\subsection{Immunoperoxidase procedure: streptavidin-biotin or avidin-biotin}

The streptavidin-biotin kit reagents (Histomark $^{\mathrm{ru}}$; Kirkegaard \& Perry, Gaithersburg, MD 20879, USA) are supplied in ready-to-use dilutions and have produced excellent results. For the $A B C$ kit (Vector Labs Vectastain $A B C^{\mathrm{TM}}$ ), the biotinylated second antibody, e.g., goat antirabbit and the avidin/biotinylated-peroxidase complex $(\mathrm{ABC})$ were prepared at twice the normal concentration.
(10) Rinse in PBS
$3 \times 3 \min$

(11) Iotinylated second antibody

$60 \mathrm{~min}$

(12) Rinse in PBS

$3 \times 3 \mathrm{~min}$

(13) ABC complex or streptavidin-

$60 \mathrm{~min}$ biotinylated peroxidase complex

(14) Rinse in PBS

$3 \times 3 \min$

(15) DAB histochemistry: nickel-cobalt 3-10 min intensified (Adams, 1981)

(16) Rinse in PBS

$3 \times 3 \min$

(17) Dehydrate and clear: $50 \%, 70 \%, 95 \%, 1 \mathrm{~min}$ in each $100 \%$ EtOH, xylenes $\times 2$

(18) Coverslip with Permount (sections can be air-dried and counterstained with toluidine blue if desired before coverslipping). 


\section{Results and discussion}

Previous methods of flat-embedding have used a sandwich approach, trapping tissue slabs between layers of plastic or other surfaces. We offer two technical improvements. First, tissue distortion is minimized by osmicating, dehydrating, and infiltrating the slabs with epoxy while they are held flat by the surface tension between the filter paper circles. This also reduces physical handling of the fragile tissue, thereby minimizing incidental damage. Second, because the tissue slabs are polymerized onto an epoxy slide, they form a durable, archival embedment. We
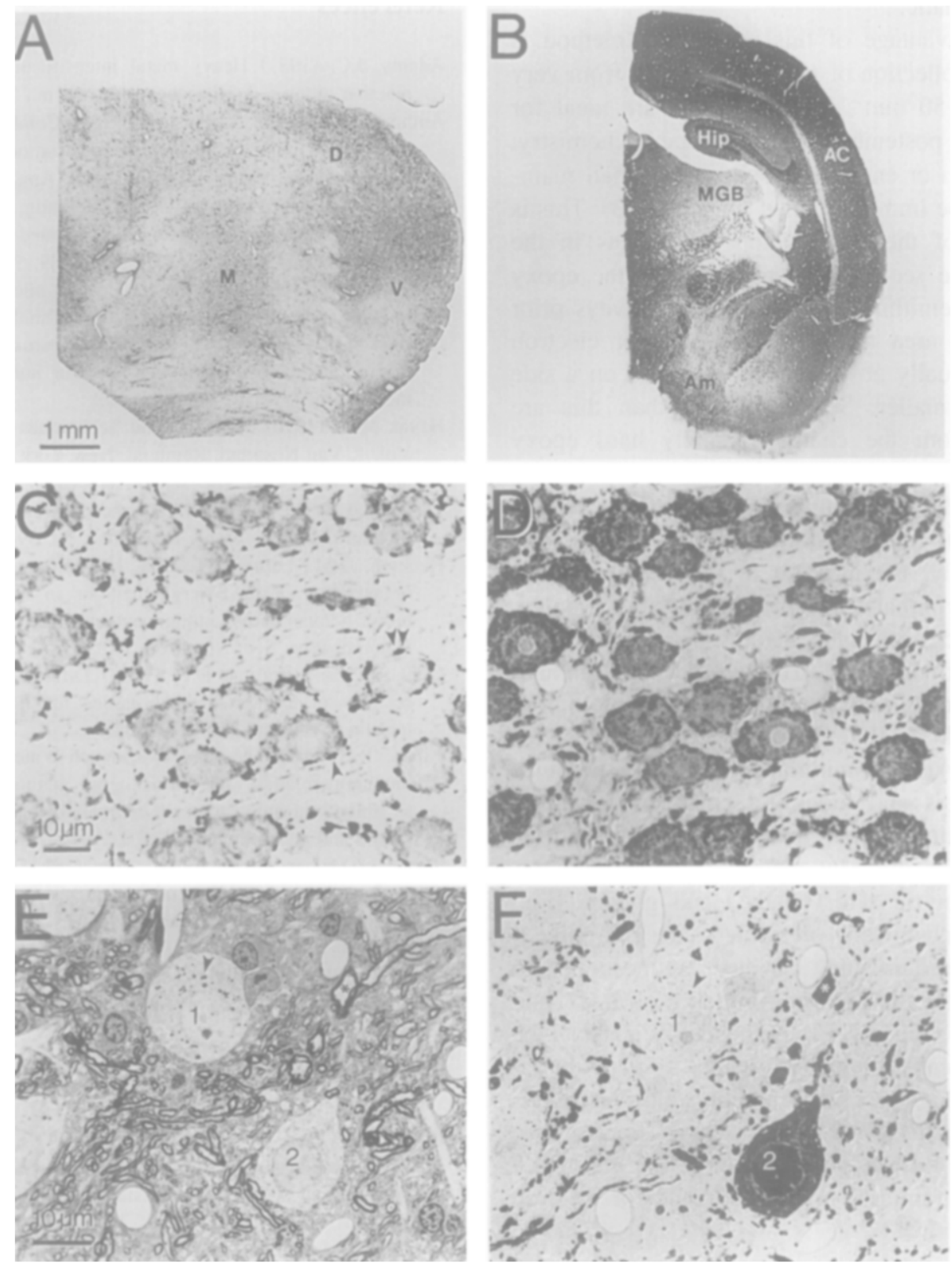

Fig. 2. Immunostained semithin sections cut with glass knives from large, flat embedded blocks. A: Hemithalamic preparation of the entire cat medial geniculate body (MGB) in a $1.5 \mu \mathrm{m}$ thick section, immunostained for $\gamma$-aminobutyric acid (GABA). D,V, and $M$ designate, respectively, the dorsal, ventral, and medial divisions of the MGB. B: Semithin section of one hemisphere of the mustache bat Pteronotus parnellii, at the level of the rostal MGB, GABA immunostained and counterstained with toluidine blue (same scale as in A). Hip: hippocampus; AC: auditory cortex; Am: amygdala. A,B: Planachromat, N.A. 0.04, X 16. C,D: Adjacent semithin sections from the bat's ventral nucleus of the lateral lemniscus, stained altemately for GABA (left), and for glycine (right). The glycine immunoreactive somata (D) receive GABA immunoreactive terminals or puncta (C, arrowhead). Certain puncta appear to co-localize both GABA and glycine immunoreactivity (C,D, double arrowheads). Planapochromat, N.A. 1.0, $\times 825$. E,F: Colocalization of retrogradely transported tracer (horseradish peroxidase; HRP) and neurotransmitter immunoreactivity from a section prepared as in Fig. IA. Adjacent semithin sections from the ventral division of the MGB in the cat after cortical injection of HRP. E: Toluidine blue-stained section showing HRP labeled ( 1 ; arrowhead), and unlabeled (2) cells, as well as large, myelinated axons (asterisk). F: The adjacent, GABA-stained section shows that neuron (2) is GABA immunoreactive while the netrogradely labeled cell (1) is immunonegative. There is conspicuous GABA immunoreactivity in the neuropil, including some of the largest myelinated axons (asterisk). Planapochromat, N.A. 1.4, $\times 938$. 
have successfully immunostained tissue from blocks mounted and sectioned up to 4 years after embedding. The $2 \mathrm{~mm}$ thick epoxy slide is easy to cut into blocks that remain flat when mounted for semithin sectioning. The other methods produce a wafer-thin, comparatively fragile embedment that must be carefully stripped away from the plastic foils or coverslips, making recovery and mounting of the slab problematic.

The primary advantage of this embedding method is that it allows the collection of semithin sections from very large areas, up to $50 \mathrm{~mm}^{2}$. These sections are ideal for global analyses in postembedding immunocytochemistry; indeed, large nuclei or entire hemispheres of small mammalian brains can be immunostained (Fig. 2A-B). This is possible because of the preservation of flatness in the embedment and the sectioning consistency of the epoxy resin. Traditional semithin sections, used as surveys prior to selection of an area for thin sectioning for electron microscopy, are usually no more than $2-3 \mathrm{~mm}$ on a side and often much smaller. Sections larger than this are difficult to cut from the characteristically hard epoxy preparations because of damage to the glass knives. Our epoxy recipe was derived from earlier work (Parsons and Darden, 1961) with an adjustment in the ratio of the components, notably, the increase in the amount of the plasticizer, dibutyl phthalate, from $4 \%$ to $10 \%$ (Grimley, 1965). This produces an embedment sufficiently hard to cut even 'gold' sections ( $\sim 0.1 \mu \mathrm{m}$ thick), yet soft enough to permit dozens of semithin sections to be cut before degradation of the knife edge. This provides flexibility for serial section analysis and minimizes loss of tissue since fewer knife changes and block refacings are required. Adjacent serial sections can be mounted on the same slide and then stained for different neurotransmitter antigens, such as $\gamma$-aminobutyric acid (GABA) and glycine (Fig. $2 \mathrm{C}, \mathrm{D})$. When histochemically prereacted slabs are embedded using this procedure, they can be immunostained to reveal colocalization of neurotransmitter molecules and transported tracers (Fig. 2E,F). Embedded slabs can be studied microscopically as whole mounts to pinpoint labeled cells or as a high-resolution myelin stain. While this method was originally intended for light microscopic preparations, with further polymerization the plastic is compatible with ultramicrotomy and suitable for electron microscopy.

\section{Acknowledgements}

We thank Dr. R.L. Saint Marie for his helpful advice in postembedding immunocytochemistry, and Dr. R.J. Wenthold for generously supplying antisera to GABA and glycine. This research was supported by United States
Public Health Service Grant RO1 DC02319-15, University of California Faculty Research Awards (J.A.W.), and by University of California Professional Development Awards (D.T.L.). Preliminary accounts have appeared (Huchton et al., 1991; Winer et al., 1995).

\section{References}

Adams, J.C. (1981) Heavy metal intensification of DAB-based HRP reaction product. J. Histochem. Cytochem., 29: 775.

Anthony, E.L.P. and King, J.C. (1986) Combined light and electron microscope immunocytochemical localization of scattered peptidergic neurons in the central nervous system. Am. J. Anat., 175: 175-195.

Erlandsen, S.L., Parsons, J.A. and Rodning, C.B. (1979) Technical parameters of immunostaining of osmicated tissue in epoxy sections. J. Histochem. Cytochem., 27: 1286-1289.

Grimley, P.M. (1965) Selection for electron microscopy of specific areas in large epoxy tissue sections. Stain Technol., 40: 259-263.

Hancock, M.B. (1986) Two-color immunoperoxidase staining: visualization of anatomic relationships between immunoreactive neural elements. Am. J. Anat., 175: 343-352.

Hayat, M.A. (1970) Principles and Techniques of Electron Microscopy, Vol. 1, Van Nostrand, Reinhold, New York, pp. 109-177.

Holländer, H. (1970) The section embedding (se) technique. A new method for the combined light and electron microscopic examination of central nervous tissue. Brain Res., 20: 39-47.

Huchton, D.M., Lanue, D.T., Sun, J.Y.-M. and Winer, J.A. (1991) The organization of GABAergic neurons in the cat medial geniculate body: a quantitative immunocytochemical study of post-embedded material. Proc. Soc. Neurosci., 17: 300.

Lane, B.P. and Europa, D.L. (1965) Differential staining of ultrathin sections of Epon-embedded tissues for light microscopy. J. Histochem. Cytochem., 13: 579-582.

Papka, R.E., Yu, S.M. and Nikitovitch-Winer, M.B. (1986) Use of immunopcroxidase and immunogold methods in studying prolactin secretion and application of immunogold labeling for pituitary hormones and neuropeptides. Am. J. Anat., 175: 289-306.

Parsons, D.F. and Darden, Jr., E.B. (1961) Optimal conditions for methacrylate embedding of certain tissues and cells sensitive to polymerization damage. Exp. Cell Res., 24: 466-483.

Saint Marie, R.L., Ostapoff, E.-M., Morest, D.K. and Wenthold, R.J. (1989) Glycine immunoreactive projections of the cat lateral superior olive: possible role in midbrain ear dominance. J. Comp. Neurol., 279: 382-396.

Schwartz, I.R. (1982) A simple technique for osmicating and flat cmbedding large tissue sections for light and electron microscopy. Stain Technol., 57: 52-54.

Wilson, C.J. and Groves, P.M. (1979) A simple and rapid section embedding technique for sequential light and electron microscopic examination of individually stained central neurons. J. Neurosci. Methods, 1: 383-391.

Winer, J.A., Larue, D.T. and Pollak, G.D. (1995) GABA and glycine in the central auditory system of the mustache bat: structural substrates for inhibitory neuronal organization. J. Comp. Neurol., 355: 317-353.

Yingcharoen, K., Rinvik, J., Storm-Mathisen, J. and Ottersen, O.P. (1989) GABA, glycine, glutamate, aspartate and taurine in the perihypoglossal nuclei: an immunocytochemical investigation in the cat with particular reference to the issue of amino acid colocalization. Exp. Brain Res., 78: 345-357.

Yu, S.M. and Schwartz, I.R. (1989) An improved flat embedding technique for immunoelectron microscopy. Stain Technol., 64: 143-146. 